Mathematical Research Letters 12, 269-274 (2005)

\title{
LAPLACIANS ON FRACTALS WITH SPECTRAL GAPS HAVE NICER FOURIER SERIES
}

\author{
Robert S. StRICHARTZ
}

\begin{abstract}
On the Sierpinski gasket and related fractals, partial sums of Fourier series (spectral expansions of the Laplacian) converge along certain special subsequences. This is related to the existence of gaps in the spectrum.
\end{abstract}

Laplacians on fractals have been studied intensively using both probabilistic and analytic tools, as a "rough" counterpart to Laplacians on smooth Riemannian manifolds ([B], [Ki], [S1]). This research has succeeded in establishing many "expected" analogs of results from the smooth theory, but has also turned up some startling differences. For example: there exist localized eigenfunctions [FS]; the square of a nonconstant function in the domain of the Laplacian is never in the domain of the Laplacian [BST]; the energy measure is singular $[\mathrm{Ku}]$; the wave equation has infinite propagation speed [DSV]; the Weyl ratio does not have a limit $[\mathrm{FS}],[\mathrm{KL}]$; the Laplacian does not behave like a second order operator [S2]; to mention just a few. One might be tempted to say that the fractal world resembles the smooth world to some degree, but everything is worse.

On the other hand, recent numerical experiments hint that when it comes to convergence of Fourier series, things might be better on fractals. To be specific, consider the standard Laplacican $\Delta$ on the Sierpinski gasket SG. With either Dirichlet or Neumann boundary conditions, there is a complete orthonormal basis of eigenfunctions, say $-\Delta u_{j}=\lambda_{j} u_{j}, j=1,2,3, \ldots$, and every $L^{2}$ function $f$ has a Fourier series

$$
f=\sum_{j=1}^{\infty} c_{j} u_{j} \text { with } c_{j}=\int_{S G} f u_{j} d \mu,
$$

the analog of Fourier sine and cosine series on the interval. The partial sums

$$
S_{N} f(x)=\sum_{j=1}^{N} c_{j} u_{j}(x)
$$

Received May 10, 2004.

Research supported in part by the National Science Foundation, grant DMS-0140194.

Key words and phrases. Analysis on fractals, Sierpinski gasket, Fourier series on fractals

2000 Mathematics Subject Classification. Primary 28A80, 42C99 
may be expresed as an integral

$$
S_{N} f(x)=\int_{S G} D_{N}(x, y) f(y) d \mu(y)
$$

against a Dirichlet kernel

$$
D_{N}(x, y)=\sum_{j=1}^{N} u_{j}(x) u_{j}(y) .
$$

Because many eigenvalues have nontrivial multiplicities, it does not make sense to allow all values of $N$, but only those that do not split multiple eigenspaces. Even among these allowable values, some are more natural than others. In [OSS], computations of $D_{N}$ for these natural choices (for small values of $N$ ) showed a clear approximate identity behavior. This stands in striking contrast to the wooly behavior of the usual Dirichlet kernel, and leads to the hope that for continuous functions, $S_{N} f$ might converge uniformly to $f$ along an appropriate subsequence. In this note we will provide a proof of this fact. Also, in [CDS] it was observed numerically that for some functions with a jump discontinuity, $S_{N} f$ exhibits a Gibbs' phenomenon, but not as strong as the usual one. This means that

$$
\int\left|D_{N}(x, y)\right| d \mu(y)
$$

is uniformly bounded, but does not converge to 1 .

The key observation is that the spectrum of the Laplacian on SG and other fractals is very "lumpy". One manifestation of this is the occurrence of large gaps. Also, the particular partial sums $S_{N} f$ that we consider extend up to the beginning of a gap. This means that the partial sum is equal to an approximation obtained by using a summability method

$$
\sigma_{N} f(x)=\sum_{j=1}^{\infty} \psi\left(\lambda_{j} / \lambda_{N}\right) c_{j} u_{j}(x)
$$

where $\psi$ is a smooth cutoff function satisfying $\psi(t)=1$ for $t \leq 1$ and $\psi(t)=0$ for $t \geq \lambda_{N+1} / \lambda_{N}$. Note that we require a uniform lower bound for $\left(\lambda_{N+1} / \lambda_{N}\right)-1$ in order to use the same cut-off function $\psi$. It is then not too surprising that the summability method is well-behaved.

Theorem 1. a) Let $\left\{N_{m}\right\}$ be a sequence of integers such that $\left(\lambda_{N_{m}+1} / \lambda_{N_{m}}\right)-1$ is bounded away from zero. Then $S_{N_{m}} f \rightarrow f$ as $m \rightarrow \infty$ in $L^{q}$ if $f \in L^{q}$, $1 \leq q<\infty$, and uniformly if $f$ is continuous. 
b) There exist infinitely many disjoint sequences $\left\{N_{m}\right\}$ such that $\lambda_{N_{m}+1} / \lambda_{N_{m}}=$ $C>1$. One example is $N_{m}=\frac{1}{2}\left(3^{m+1}-3\right)$ (Dirichlet) or $N_{m}=\frac{1}{2}\left(3^{m+1}+3\right)$ (Neumann). There is also an example with $C>2$.

Proof: The details are easy because all the work has already been done. An exact description of the spectrum (Dirichlet and Neumann) of the Laplacian on SG was given by Fukushima and Shima [FS] using analytic methods (spectral decimation). The spectral gaps are implicit in this work, and are stated explicitly in Theorem 5.1 of [GRS]. The "natural" choices for $N$ in [DSS] are $N_{m}=\frac{1}{2}\left(3^{m+1}-3\right)$ for Dirichlet and $N_{m}=\frac{1}{2}\left(3^{m+1}+3\right)$ for Neumann boundary conditions, for $m \geq 2$, since these are the number of (nonboundary) vertices in the $m$ th graph approximation to SG. The corresponding eigenvalues are $\lambda_{N_{m}}=$ $5^{m-2} \lambda_{1}^{(6)}$ and $\lambda_{N_{m}+1}=5^{m-1} \lambda_{1}^{(5)}$ where $\lambda_{1}^{(5)} \approx 172.364$ and $\lambda_{1}^{(6)} \approx 677.859$ (using notation of [GRS] and [DSV]). It follows that the gap ratio $\lambda_{N_{m}+1} / \lambda_{N_{m}}$ is always the same, $5 \lambda_{1}^{(5)} / \lambda_{1}^{(6)} \approx 1.271$. In fact there are infinitely many choices of sequences $\lambda_{N}$ such that $\lambda_{N}$ and $\lambda_{N+1}$ increase by multiples of 5 , so the gap ratio is constant. In fact the biggest gap ratio occcurs if we choose the eigenvalue $5^{m-1} \lambda_{0}^{(5)}\left(\lambda_{0}^{(5)} \approx 55.885\right)$ which occurs immediately before $\lambda_{N_{m}}$. Then the gap ratio is $\lambda_{1}^{((6)} / 5 \lambda_{0}^{(5)} \approx 2.425$. All these eigenvalues have high multiplicities; in fact the multiplicity grows exponentially with $m$ as $m \rightarrow \infty$. This undoubtedly is related to the existence of the gaps, although a direct argument is not available.

The next ingredient in the argument is a "generic" spectral multiplier theorem of Duong, Ouhabaz and Sikora ([DOS], Theorem 6.2), which yields $\sigma_{N} f \rightarrow f$ in $L^{q}$ if $f \in L^{q}, 1 \leq q<\infty$, and $\sigma_{N} f \rightarrow f$ uniformly if $f$ is continuous. In order to verify the hypothesis of that theorem (with $p=\infty$ ), the essential requirement is the appropriate heat kernel estimates. These were originally established for SG by Barlow and Perkins $[\mathrm{BP}]$, and have since been extended to a variety of fractals (se $[\mathrm{BB}]$ and $[\mathrm{HK}]$, for example). All proofs of heat kernel estimates on fractals use probabilistic methods.

A related result is the $L^{q}$ boundedness, above and below, for $1<q<\infty$, of the Littlewood-Paley function:

Theorem 2. Let

$$
S f(x)=\left(\sum_{m=1}^{\infty}\left|S_{m} f(x)\right|^{2}\right)^{1 / 2}
$$

for

$$
S_{m} f(x)=\sum_{j=N_{m-1}+1}^{N_{m}} c_{j} u_{j}(x)
$$


where $\left\{N_{m}\right\}$ is any sequence as in Theorem 1 b). Then $A_{q}\|f\|_{q} \leq\|S f\|_{q} \leq$ $B_{q}\|f\|_{q}$ for $1<q<\infty$.

Proof: The Rademacher function argument given in Stein [St] Chapter 4, section 5 , requires the uniform boundedness in $L^{q}$ of $\sum_{m=1}^{\infty} \varepsilon_{m} S_{m} f(x)$ for any choice of $\varepsilon_{m}= \pm 1$. Once again we can write

$$
S_{m} f(x)=\sum_{j=1}^{\infty} \widetilde{\psi}\left(\lambda_{j} / \lambda_{N_{m}}\right) c_{j} u_{j}(x)
$$

for the appropriate smooth cut-off function $\widetilde{\psi}$, and so $\sum_{m=1}^{\infty} \varepsilon_{m} S_{m} f(x)$ is a spectral multiplier operator with multiplier $\sum_{m=1}^{\infty} \varepsilon_{m} \widetilde{\psi}\left(\lambda_{j} / \lambda_{N_{m}}\right)$ satisfying a Hörmandertype condition with bounds independent of the choice of $\left\{\varepsilon_{m}\right\}$. Then Theorem 3.1 (with $p=\infty$ ) of [DOS] gives the desired bounds on the spectral multiplier operator.

The same results should be valid for many other fractals. For post-critically finite (pcf) fractals for which the spectral decimation method is valid ([Sh]), one can use essentially the same reasoning. A larger class of pcf fractals, those with large enough symmetry groups, has been shown to have localized eigenfunctions associated with eigenvalues of high multiplicity $[\mathrm{BK}]$. It is reasonable to expect that these examples will also have the required spectral gaps. One such example, the pentagasket, which does not allow spectral decimation, has been examined in detail in $[\mathrm{ASST}]$. Numerical data presented there (more data is available on the

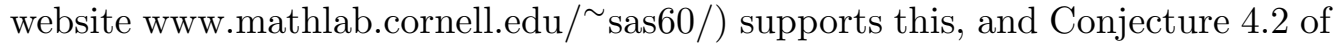
[ASST] shows exactly where in the spectrum to find the gaps. Again there is a pair of consecutive eigenvalues $\lambda_{N}, \lambda_{N+1}$, with ratio $\approx 1139.548 / 520.308 \approx 2.190$ greater than 2 , and $\rho^{m} \lambda_{N}, \rho^{m} \lambda_{N+1}$, are also eigenvalues for every positive integer $m$, for $\rho=40 /(\sqrt{161}-9) \approx 10.84289$. The only thing that remains to be shown is that the pairs $\rho^{m} \lambda_{N}, \rho^{m} \lambda_{N+1}$, are always consecutive eigenvalues.

Another class of examples are the compact fractafolds based on SG, described in [S3]. Basically, these are obtained by taking a finite number of copies of SG and identifying certain pairs of boundary points. A complete description of the spectrum of the Laplacian is given in Theorem 4.3 (in the case of no boundary) and Theorem 5.1 (Dirichlet and Neumann boundary conditions) of [S3], and the identical spectral gaps as for SG appear.

Products of SG are discussed in [S4]. These are self-similar fractals that are not pcf, but it is easy enough to understand the spectral theory of the 
Laplacian as a tensor product of the single SG: For the double product $S G^{2}$, the eigenfunctions are $u_{j} \otimes u_{k}$ with eigenvalues $\lambda_{j}+\lambda_{k}$. It is easy to see that the "square" partial sums

$$
S_{N_{m}} f=\sum_{j=1}^{N_{m}} \sum_{k=1}^{N_{m}} c_{j, k} u_{j} \otimes u_{k}
$$

will have the same convergence properties as in the single SG case (the associated Dirichlet kernel will just be the tensor product of single SG Dirichlet kernels). Of course the more natural "circular" partial sums would be of the form

$$
\widetilde{S}_{M} f=\sum_{\lambda_{j}+\lambda_{k} \leq M} c_{j, k} u_{j} \otimes u_{k} .
$$

But if we choose $N_{m}$ so that the ratio of the eigenvalues $\left(\lambda_{N_{m}}+1\right) / \lambda_{N_{m}}$ exceeds 2 , then $S_{N_{m}}=\widetilde{S}_{2 \lambda_{N_{m}}}$. Of course this coincidence does not occur for products of 3 or more copies of SG, since no ratio exceeds 3 . But the same argument could be applied to the product of 2 copies of the pentagasket.

Another interesting non-pcf example is the Sierpinski carpet. The required heat kernel estimates are known $[\mathrm{BB}]$, but nothing is known about the structure of the spectrum of the Laplacian.

Another interesting consequence of spectral gaps will be reported in [BS] (see

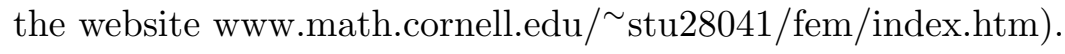

\section{Note added in proof}

In a recent preprint "Convergence of Mock Fourier Series", we give another example of a fractal analog of Fourier Series (with respect to certain Cantor measures) that enjoys similar convergence properties.

\section{Acknowledgment}

I am grateful to Adam Sikora for a detailed explanation of his joint paper [DOS].

\section{References}

[ASST] B. Adams, S. A. Smith, R. Strichartz and A. Teplyaev, The spectrum of the Laplacian on the pentagasket, Trends in Mathematics, Fractals in Graz 2001, Birkhauser (2003), 1-24.

[B] M. Barlow, Diffusion on fractals, Lecture Notes Math., vol. 1690, Springer, 1998.

[BB] M. T. Barlow and R. F. Bass, Brownian motion and harmonic analysis on Sierpinski carpet, Canad. J. Math. 51 (1999), 673-744.

[BK] M. Barlow and J. Kigami, Localized eigenfunctions of the Laplacian on p.c.f. selfsimilar sets, J. London Math. Soc. 56 (1997), 320-332. 
[BP] M. Barlow and E. Perkins, Brownian motion on the Sierpinski gasket, Probab. Theory Related Fields 79 (1988), 543-623.

[BST] O. Ben-Bassat, R. Strichartz and A. Teplyaev, What is not in the domain of the Laplacian on Sierpinski gasket type fractals, J. of Functional Analysis 166 (1999), 197-217.

[BS] B. Bockelman and R. Strichartz, in preparation.

[CDS] K. Coletta, K. Dias and R. Strichartz, Numerical analysis on the Sierpinski gasket, with applications to Schrödinger equations, wave equation, and Gibbs' phenomenon, Fractals. 12 (2004), 413-449.

[DSV] K. Dalrymple, R. Strichartz and J. Vinson, Fractal differential equations on the Sierpinski gasket, J. Fourier Anal. Appl. 5 (1999), 203-284.

[DOS] X. T. Duong, E. M. Ouhabaz and A. Sikora, Plancherel type estimates and sharp spectral multipliers, J. Funct. Anal. 196 (2002), 443-485.

[FS] M. Fukushima and T. Shima, On a spectral analysis for the Sierpinski gasket, Potential Anal. 1 (1992), 1-35.

[GRS] M. Gibbons, A. Raj and R. Strichartz, The finite element method on the Sierpinski gasket, Constructive Approx. 17 (2001), 561-588.

[HK] B. Hambly and T. Kumagai, Transition density estimates for diffusion processes on post critically finite self-similar fractals, Proc. London Math. Soc. 78 (1999), 431-458.

[Ki] J. Kigami, Analysis on Fractals, Cambridge University Press, New York, 2001.

[KL] J. Kigami and M. L. Lapidus, Weyl's problem for the spectral distribution of Laplacians on p.c.f. self-similar fractals, Comm. Math. Phys. 158 (1993), 93-125.

[Ku] S. Kusuoka, Dirichlet forms on fractals and products of random matrices, Publ. Res. Inst. Math. Sci. 25 (1989), 659-680.

[OSS] R. Oberlin, B. Street and R. Strichartz, Sampling on the Sierpinski gasket, Experimental Math. 12 (2003), 403-418.

[St] E. M. Stein, Singular Integrals and Differentiability Properties of Functions, Princeton University Press, Princeton, NJ, 1970.

[Sh] T. Shima, On eigenvalue problems for Laplacians on p.c.f. self-similar sets, Japan J. Indust. Appl. Math. 13 (1996), 1-23.

[S1] R. Strichartz, Analysis on fractals, Notices Amer. Math. Soc. 46 (1999), 1199-1208.

[S2] _ Function spaces on fractals, J. Funct. Anal. 198 (2003), 43-83.

[S3] - Fractafolds based on the Sierpinski gasket and their spectra, Trans. Amer. Math. Soc. 355 (2003), 4019-4043.

[S4] _ Analysis on products of fractals, Trans. Amer. Math. Soc. 357 (2005), 571615.

Mathematics Department, Malott Hall, Cornell University, IthacA, NY 14853

E-mail address: str@math.cornell.edu 